\title{
On the strict monotonicity of spectral radii for classes of bounded positive linear operators
}

\author{
Chris Guiver $^{1}{ }^{1 D}$
}

Received: 28 June 2017 / Accepted: 8 February 2018 / Published online: 24 February 2018

(C) The Author(s) 2018. This article is an open access publication

\begin{abstract}
Strict monotonicity of the spectral radii of bounded, positive, ordered linear operators is investigated. It is well-known that under reasonable assumptions, the spectral radii of two ordered positive operators enjoy a non-strict inequality. It is also well-known that a "strict" inequality between operators does not imply strict monotonicity of the spectral radii in general-some additional structure is required. We present a number of sufficient conditions on both the cone and the operators for such a strict ordering to hold which generalise known results in the literature, and have utility in comparison arguments, ubiquitous in positive systems theory.
\end{abstract}

Keywords Comparison argument - Ordered Banach space · Positive linear operator · Spectral radius

Mathematics Subject Classification 06A06 - 15B48 - 47A10 - 47A63 - 47B65 · $47 \mathrm{~N} 60 \cdot 47 \mathrm{~N} 70$

\section{Introduction}

We investigate the following problem pertaining to the spectral radii of bounded positive linear operators which admit a certain ordering. Here, and throughout the manuscript, $\mathcal{X}$ denotes a real Banach space, with positive cone $\mathcal{K} \subset \mathcal{X}$ which induces the partial order $\leq$ or $\geq$ and $A_{1}, A_{2} \in \mathcal{B}(\mathcal{X})$ denote bounded, positive linear operators with $r\left(A_{1}\right)>0$. Given these hypotheses, we seek to investigate when

Chris Guiver

c.guiver@bath.ac.uk

1 Department of Mathematical Sciences, University of Bath, Bath, UK

Birkhäuser 


$$
A_{2} \leq A_{1} \quad \text { and } \quad A_{2} \neq A_{1} \Rightarrow r\left(A_{2}\right)<r\left(A_{1}\right)
$$

holds. It is known in the finite-dimensional case that irreducibility of $A_{2}$ is sufficient for (1.1), see [22, Theorem 9]. For general cones, it is known that if $\mathcal{K}$ is normal and reproducing, then

$$
A_{2} \leq A_{1} \Rightarrow r\left(A_{2}\right) \leq r\left(A_{1}\right)
$$

(see, for example, [19, Theorem 4.2] or [5, Theorem 1.1]). The non-strict inequality (1.2) has been considered for more general cones and positive operators in [5], which builds on earlier work such as [23], and shown not to hold in general when the assumptions of normality or reproducing are dropped, and not replaced with suitable alternatives. The strict inequality (1.1) has been considered in [19], and sufficient conditions given. By way of further background, we mention that there is also a body of work on monotonicity of the spectral radius for commuting ordered Banach algebras, see [21] and the references therein.

The trivial example wherein $\mathcal{X}=\mathbb{R}^{2}, \mathcal{K}=\mathbb{R}_{+}^{2}$ (which is normal and reproducing), with the usual partial ordering of componentwise inequality, and

$$
A_{2}=\left(\begin{array}{ll}
1 & 0 \\
0 & 1
\end{array}\right) \text { and } A_{1}=\left(\begin{array}{ll}
1 & x \\
0 & 1
\end{array}\right) \quad x>0
$$

shows that (1.1) need not hold in general. As another example, consider the bounded linear operators

$$
A_{2}\left(x_{1}, x_{2}, \ldots\right)=\left(0, x_{1}, x_{2}, \ldots\right) \text { and } A_{1}\left(x_{1}, x_{2}, \ldots\right)=\left(x_{1}, x_{1}, x_{2}, \ldots\right) \text {, }
$$

defined on the space of convergent sequences with zero limit, equipped with the supremum norm, and positive cone consisting of component wise nonnegative sequences. Clearly $A_{2} \leq A_{1}, A_{2} \neq A_{1}$, but both $A_{2}$ and $A_{1}$ are isometries and so $r\left(A_{1}\right)=$ $r\left(A_{2}\right)=1$.

We acknowledge that there are elementary sufficient conditions for (1.1), such as if $\mathcal{K}$ is normal and reproducing and

$$
\exists \rho>0: A_{2}+\rho I \leq A_{1} \Rightarrow r\left(A_{2}\right)<r\left(A_{1}\right),
$$

or

$$
\exists \gamma \in(0,1): A_{2} \leq \gamma A_{1} \Rightarrow r\left(A_{2}\right)<r\left(A_{1}\right) .
$$

Both (1.4) and (1.5) follow from straightforward adjustments to (1.2), using the known equalities $r\left(A_{2}+\rho I\right)=r\left(A_{2}\right)+\rho$ and $r\left(\gamma A_{1}\right)=\gamma r\left(A_{1}\right)$. The assumptions in (1.4) and (1.5) are too conservative for many applications, however. We are interested in the strict inequality (1.1) owing to its utility for discrete-time positive dynamical systems, where comparison arguments are readily applicable, such as [10], particularly in the infinite-dimensional case. For example, much attention has been devoted in theoretical ecology to discrete-time dynamical systems specified by certain classes of integral operators, so-called Integral Projection Models [7,8]. Here the spectral radius gives a theoretical long-term exponential growth (or decline) rate of a population. 
There is considerable overlap between the present work and aspects of [19], where (1.1) is also considered. Briefly, we derive sufficient conditions for (1.1) which are distinct to those in [19] and we highlight the differences in the manuscript. Finally, we draw heavily on the textbook [12] but, to the best of our knowledge, the results presented here do not appear in [12] or indeed elsewhere in the literature.

\section{Notation and preliminaries}

There are a number of conventions pertaining to terminology in the positive operator and positive systems literature, which are not all equivalent, and we use those in [12]. We briefly recall some key terms. Let $(\mathcal{X},\|\cdot\|)$ denote a real Banach space. A (positive) cone $\mathcal{K} \subseteq \mathcal{X}$ is a closed subset of $\mathcal{X}$ such that $\mathcal{K}+\mathcal{K} \subseteq \mathcal{K}, \alpha \mathcal{K} \subseteq \mathcal{K}$ for all $\alpha \geq 0$ and $\mathcal{K} \cap(-\mathcal{K})=\{0\}$. The cone is called reproducing (also sometimes known as generating) if $\mathcal{X}=\mathcal{K}-\mathcal{K}$ and normal if $0 \leq x \leq y$ implies that $\|x\| \leq a\|y\|$ for some constant $a>0$ which is independent of $x$ and $y$. A cone is called solid if it has non-empty interior. Solid cones are reproducing.

For $u \in \mathcal{K} \backslash\{0\}$ we shall require the set

$$
\mathcal{X}_{u}:=\{x \in \mathcal{X}:-\gamma u \leq x \leq \gamma u \text {, for some } \gamma \geq 0\}
$$

(see [12, p. 42]). It is clear that $u \in \mathcal{X}_{u}$ and hence $\mathcal{X}_{u} \neq \emptyset$. Furthermore, as $\mathcal{X}_{u}$ is closed under addition and scalar multiplication, it follows that $\mathcal{X}_{u} \subseteq \mathcal{X}$ is a subspace. Thus $\mathcal{X}_{u}$ is a normed space when equipped with

$$
\|x\|_{u}:=\inf \{\gamma \geq 0:-\gamma u \leq x \leq \gamma u\}, \quad x \in \mathcal{X}_{u},
$$

which has the elementary properties:

i. $\|u\|_{u}=1$

ii. for $x, y \in \mathcal{X}_{u} \cap \mathcal{K}, x \leq y$ implies that $\|x\|_{u} \leq\|y\|_{u}$;

iii. for $x \in \mathcal{X}_{u},-\|x\|_{u} u \leq x \leq\|x\|_{u} u$.

For Banach spaces $\mathcal{X}$ and $\mathcal{Y}$, we let $\mathcal{B}(\mathcal{X}, \mathcal{Y})$ and $\mathcal{B}(\mathcal{X})$ denote the set of bounded linear operators $\mathcal{X} \rightarrow \mathcal{Y}$ and $\mathcal{X} \rightarrow \mathcal{X}$, respectively. We let $\mathcal{C}(\mathcal{X}) \subseteq \mathcal{B}(\mathcal{X})$ denote the subset of compact operators. The (continuous) dual of $\mathcal{X}$, the set of bounded, realvalued linear functionals on $\mathcal{X}$, is denoted $\mathcal{X}^{\prime}=\mathbb{B}(\mathcal{X}, \mathbb{R})$ and equipped with usual norm

$$
\|f\|_{\mathcal{X}^{\prime}}=\sup _{\substack{x \in \mathcal{X} \\ x \neq 0}} \frac{|f(x)|}{\|x\|} .
$$

Given $A \in \mathcal{B}(\mathcal{X})$, we recall that the adjoint operator $A^{\prime} \in \mathcal{B}\left(\mathcal{X}^{\prime}\right)$ is defined by $\left(A^{\prime} f\right)(x)=f(A x)$ for all $f \in \mathcal{X}^{\prime}$ and all $x \in \mathcal{X}$ (see, for example, [13, Definition $4.5-1$, p. 232]). The adjoint operator $A^{\prime}$ is bounded with respect to the induced operator norm 


$$
\left\|A^{\prime}\right\|_{\mathcal{X}^{\prime}}=\sup _{\substack{f \in \mathcal{X}^{\prime} \\ f \neq 0}} \frac{\left\|A^{\prime} f\right\|_{\mathcal{X}^{\prime}}}{\|f\| \mathcal{X}^{\prime}}
$$

If $\mathcal{Y}$ has cone $\mathcal{L}$, then the operator $A \in \mathcal{B}(\mathcal{X}, \mathcal{Y})$ is called positive if $\mathcal{A K} \subseteq \mathcal{L}$. A positive operator $A \in \mathcal{B}(\mathcal{X})$ is called $u$-bounded if there exist functions $\alpha, \beta: \mathcal{X} \rightarrow$ $\mathbb{R}_{+}$such that

$$
\alpha(x) u \leq A x \leq \beta(x) u \quad \forall x \in \mathcal{K},
$$

and $\alpha(x), \beta(x)>0$ if $x \neq 0$. The term $u$-bounded from above means that only the second inequality in (2.1) holds. If $\mathcal{K}$ is reproducing and $A \in \mathcal{B}(\mathcal{X})$ is a positive operator which is $u$-bounded from above, then $A: \mathcal{X} \rightarrow \mathcal{X}_{u}$ is well-defined. Moreover, $A \mid \mathcal{X}_{u} \in \mathcal{B}\left(\mathcal{X}_{u}\right)$ and the induced operator norm satisfies

$$
\|A\| \mathcal{X}_{u}=\sup _{\substack{x \in \mathcal{X}_{u} \\ x \neq 0}} \frac{\|A x\|_{u}}{\|x\|_{u}}=\|A u\|_{\mathcal{X}_{u}}
$$

The above claims are all easily established from their definitions.

We shall make use of the following properties without further reference. First, if the three conditions all hold: (i) $\mathcal{W} \subseteq \mathcal{X}$ is continuously embedded in $\mathcal{X}$; (ii) $A \in \mathcal{B}(\mathcal{X})$ has positive spectral radius, and; (iii) additionally $A \in \mathcal{B}(\mathcal{X}, \mathcal{W})$, then the spectral radii of $\mathcal{A} \in \mathcal{B}(\mathcal{X})$ and $\left.\mathcal{A}\right|_{\mathcal{W}} \in \mathcal{B}(\mathcal{W})$ are equal.

Second, if $\mathcal{X}=\overline{\mathcal{K}-\mathcal{K}}, A_{1}, A_{2} \in \mathcal{B}(\mathcal{X}), A_{1} \leq A_{2}$ and $A_{1} \neq A_{2}$, then there exists $x^{*} \in \mathcal{K}$ such that

$$
\left(A_{1}-A_{2}\right) x^{*} \neq 0,
$$

which is readily established by contraposition. Indeed, if (2.3) fails, that is,

$$
\left(A_{1}-A_{2}\right) v=0 \quad \forall v \in \mathcal{K}
$$

then, for arbitrary $x \in \mathcal{X}$, there exists $\left(u_{n}\right)_{n \in \mathbb{N}} \subset \mathcal{K},\left(v_{n}\right)_{n \in \mathbb{N}} \subset \mathcal{K}$ such that

$$
x=\lim _{n \rightarrow \infty}\left(u_{n}-v_{n}\right) .
$$

Thus, by (2.4) and continuity of $A_{1}$ and $A_{2}$

$$
\begin{aligned}
A_{1} x & =A_{1} \lim _{n \rightarrow \infty}\left(u_{n}-v_{n}\right)=\lim _{n \rightarrow \infty}\left(A_{1} u_{n}-A_{1} v_{n}\right)=\lim _{n \rightarrow \infty}\left(A_{2} u_{n}-A_{2} v_{n}\right) \\
& =A_{2} \lim _{n \rightarrow \infty}\left(u_{n}-v_{n}\right)=A_{2} x
\end{aligned}
$$

implying that $A_{1}=A_{2}$.

Following the convention of [12], we say that positive $A \in \mathcal{B}(\mathcal{X})$ is irreducible if $A x \leq \kappa x$ for some $\kappa \geq 0$ and $x \in \mathcal{K} \backslash\{0\}$ implies that $x$ is a quasi-interior point of $\mathcal{K}$. Recall that $x \in \mathcal{K}$ is a quasi-interior point if $f(x)>0$ for all non-zero, positive functionals $f \in \mathcal{X}^{\prime}$. A positive $u$-bounded operator $A \in \mathcal{B}(\mathcal{X})$ is irreducible if $u$ is a quasi-interior point. We comment that for non-solid cones, the definition of quasiinterior point used here is not equivalent to that used in [23], see [12, p. 36]. If $\mathcal{K}$ 
is solid, then the sets of quasi-interior points and interior points coincide. We let $\mathcal{K}^{\prime}$ denote the set of positive functionals in $\mathcal{B}(\mathcal{X}, \mathbb{R})$, which is a positive cone if, and only if, $\mathcal{X}=\overline{\mathcal{K}-\mathcal{K}}$.

Finally, we note that the arguments which follow make assertions about the spectrum of an operator $A \in \mathcal{B}(\mathcal{X})$, and so strictly speaking we extend $A$ to the complexification of $\mathcal{X}$, denoted $\mathcal{X}_{c}$ in the usual way; see, for example [6, p.79].

\section{Strict monotonicity of spectral radii}

Our main results are contained here. The first subsection considers estimates in the spirit of (1.4) and (1.5). The second appeals to spectral properties of positive operators. For notational convenience throughout, let $r_{1}:=r\left(A_{1}\right)$ and $r_{2}:=r\left(A_{2}\right)$.

\section{Strict monotonicity of spectral radii by direct estimates}

Lemma 3.1 Let $\mathcal{X}$ denote a real Banach space, with a cone $\mathcal{K} \subset \mathcal{X}$ and positive operators $A_{1}, A_{2} \in \mathcal{B}(\mathcal{X})$ which satisfy $A_{2} \leq A_{1}$ and $A_{1} \neq A_{2}$. If either of the following:

(1) there exist $w_{2} \in \mathcal{K} \backslash\{0\}$ such that $A_{2} w_{2}=r_{2} w_{2}, \varepsilon>0$ and $n \in \mathbb{N}$ such that

$$
r_{2}^{n} w_{2} \leq\left(A_{1}-\varepsilon I\right)^{n} w_{2}
$$

or $\gamma \in(0,1)$ such that

$$
r_{2}^{n} w_{2} \leq \gamma^{n} A_{1}^{n} w_{2}
$$

(2) there exist $f_{2} \in \mathcal{K}^{\prime} \backslash\{0\}$ such that $A_{2}^{\prime} f_{2}=r_{2} f_{2}, \varepsilon>0$ and $n \in \mathbb{N}$ such that

$$
r_{2}^{n} f_{2} \leq\left(A_{1}^{\prime}-\varepsilon I\right)^{n} f_{2},
$$

or $\gamma \in(0,1)$ such that

$$
r_{2}^{n} f_{2} \leq \gamma^{n}\left(A_{1}^{\prime}\right)^{n} f_{2}
$$

hold, then $r\left(A_{2}\right)<r\left(A_{1}\right)$.

Proof We consider the hypotheses in (1). If (3.1) holds, then $r_{2}^{n} w_{2}=A_{2}^{n} w_{2} \leq\left(A_{1}-\right.$ $\varepsilon I)^{n} w_{2}$. It follows from [12, Lemma 9.1, p. 89] that

$$
r_{2}^{n} \leq r\left(\left(A_{1}-\varepsilon I\right)^{n}\right)=\left(r\left(A_{1}-\varepsilon I\right)\right)^{n}
$$

whence

$$
r\left(A_{2}\right) \leq r\left(A_{1}-\varepsilon I\right)=r\left(A_{1}\right)-\varepsilon<r\left(A_{1}\right) .
$$


If (3.2) holds instead, then we again invoke [12, Lemma 9.1, p. 89] to see that

$$
r_{2}^{n} \leq r\left(\left(\gamma A_{1}\right)^{n}\right)=\gamma^{n}\left(r\left(A_{1}\right)\right)^{n}
$$

yielding

$$
r\left(A_{2}\right) \leq \gamma r\left(A_{1}\right)<r\left(A_{1}\right)
$$

as required. The proof for (2) is identical, save using $r\left(A^{\prime}\right)=r(A)$ for $A \in \mathcal{B}(\mathcal{X})$.

Although Lemma 3.1 is appealing in that no conditions are placed on the cone $\mathcal{K}$, the difficulty with using the lemma in practice is verifying that the inequalities in (1) or (2) hold, which requires knowledge of $w_{2}$ or $f_{2}$, respectively. It is well-known that for general operators, the assumptions in (1) and (2) are not symmetric-existence of eigenfunctionals need not imply existence of eigenvectors, for instance, see Example 4.1.

Lemma 3.2 Let $\mathcal{X}$ denote a real Banach space, with a reproducing and normal cone $\mathcal{K} \subset \mathcal{X}$ and positive linear operators $A_{1}, A_{2} \in \mathcal{B}(\mathcal{X})$ which satisfy $A_{2} \leq A_{1}$ and $A_{1} \neq A_{2}$. Assume that $w_{1} \in \mathcal{K} \backslash\{0\}$ satisfies $A_{1} w_{1}=r_{1} w_{1}$ and that one of the following:

- $A_{1}$ is $w_{1}$-bounded from above;

- $A_{1}$ is u-bounded, for some $u \in \mathcal{K} \backslash\{0\}$;

hold. It follows that $r\left(A_{2}\right)<r\left(A_{1}\right)$ if, and only if, there exist $\gamma \in(0,1)$ and $N \in \mathbb{N}$ such that

$$
A_{2}^{n} w_{1} \leq\left(\gamma r_{1}\right)^{n} w_{1} \quad \forall n \in \mathbb{N}, n \geq N
$$

Clearly, $A_{2} w_{1} \leq \gamma r_{1} w_{1}$ is sufficient for (3.3) to hold with $N=1$. However, consider the simple example

$$
\mathcal{X}=\mathbb{R}^{3}, \quad \mathcal{K}=\mathbb{R}_{+}^{3}, \quad A_{2}=\left(\begin{array}{ccc}
0 & 0 & \kappa \\
1 & 0 & 0 \\
0 & 1 & 0
\end{array}\right) \quad \text { and } \quad A_{1}=\left(\begin{array}{lll}
0 & 0 & 1 \\
1 & 0 & 0 \\
0 & 1 & 0
\end{array}\right)
$$

where $\kappa \in(0,1)$. We have that $r_{2}<r_{1}=1$ and may choose $w_{1}=\left(\begin{array}{lll}1 & 1 & 1\end{array}\right)^{T}$. By considering $A_{2}^{n} w_{1}$ for $n \in \mathbb{N}$ we see that (3.3) holds for $N \geq 3$, but not for $N \in\{1,2\}$.

Proof of Lemma 3.2 The assumption that $\mathcal{K}$ is normal implies that $\mathcal{X}_{w_{1}}$ is continuously embedded in $\mathcal{X}$, as for $x \in \mathcal{X}_{w_{1}}$

$$
-\|x\|_{w_{1}} w_{1} \leq x \leq\|x\|_{w_{1}} w_{1} \quad \Rightarrow \quad 0 \leq x+\|x\|_{w_{1}} w_{1} \leq 2\|x\|_{w_{1}} w_{1} .
$$

By the reverse triangle inequality and normality, it follows that

$$
\|x\|-\|x\|_{w_{1}}\left\|w_{1}\right\| \leq\|x+\| x\left\|_{w_{1}} w_{1}\right\| \leq 2 a\|x\|_{w_{1}}\left\|w_{1}\right\|,
$$


for some $a>0$. We conclude that

$$
\|x\| \leq(2 a+1)\left\|w_{1}\right\|\|x\|_{w_{1}}
$$

as required. Our assumptions imply that $A_{1}$ (and so also $A_{2}$ ) are $w_{1}$-bounded from above. Indeed, if $A_{1}$ is $u$-bounded, then combining (2.1) with $A_{1} w_{1}=r_{1} w_{1}$, yields

$$
\alpha\left(w_{1}\right) u \leq A_{1} w_{1}=r_{1} w_{1} \Rightarrow u \leq \frac{r_{1}}{\alpha\left(w_{1}\right)} w_{1}
$$

Here we have used that $\alpha\left(w_{1}\right)>0$ as $w_{1} \neq 0$. We conclude that

$$
A_{1} x \leq \beta(x) u \leq \frac{r_{1} \beta(x)}{\alpha\left(w_{1}\right)} w_{1}=\gamma(x) w_{1} \quad \forall x \in \mathcal{K},
$$

where $\gamma(x):=r_{1} \beta(x) / \alpha\left(w_{1}\right)$.

Therefore, by an abuse of notation we consider $A_{1}, A_{2}: \mathcal{X}_{w_{1}} \rightarrow \mathcal{X}_{w_{1}}$, which are bounded operators with the same respective spectral radii as $A_{1}, A_{2} \in \mathcal{B}(\mathcal{X})$.

Assume first that $r\left(A_{2}\right)<r\left(A_{1}\right)$. It follows that $r\left(A_{2}\right)<\gamma r\left(A_{1}\right)=r\left(\gamma A_{1}\right)$, for some $\gamma \in(0,1)$. Therefore, by the Gelfand formula for the spectral radius, there exists $N \in \mathbb{N}$ such that

$$
\begin{aligned}
\left\|A_{2}^{n} w_{1}\right\|_{w_{1}}=\left\|A_{2}^{n}\right\|_{w_{1}} & \leq\left\|\left(\gamma A_{1}\right)^{n}\right\|_{w_{1}}=\left\|\left(\gamma A_{1}\right)^{n} w_{1}\right\|_{w_{1}} \\
& =\gamma^{n} r_{1}^{n}\left\|w_{1}\right\|_{w_{1}}=\gamma^{n} r_{1}^{n}, \quad \forall n \geq N .
\end{aligned}
$$

Thus, by definition of the norm on $\mathcal{X}_{w_{1}}$

$$
A_{2}^{n} w_{1} \leq\left\|A_{2}^{n} w_{1}\right\|_{w_{1}} w_{1} \leq \gamma^{n} r_{1}^{n} w_{1} \quad \forall n \geq N
$$

which is (3.3). The converse argument reverses these steps, using the property (ii).

In its simplest form Lemma 3.2 requires verifying that inequality $A_{2} w_{1} \leq \gamma r_{1} w_{1}$, for some $\gamma \in(0,1)$ and where $w_{1}$ satisfies $A_{1} w_{1}=r_{1} w_{1}$. More assumptions are placed on the cone than in Lemma 3.1. Moreover, an obvious corollary follows by applying Lemma 3.2 to the adjoint operator, which we state next, and crucially use that normal and reproducing are dual notions between $\mathcal{K}$ and $\mathcal{K}^{\prime}$, see [12, Theorems 4.5, 4.6, p. 40].

Corollary 3.3 Imposing the notation and assumptions of Lemma 3.2, assume that $f_{1} \in \mathcal{K}^{\prime} \backslash\{0\}$ satisfies $A_{1}^{\prime} f_{1}=r_{1} f_{1}$ and that one of the following:

- $A_{1}^{\prime}$ is $f_{1}$-bounded from above;

- $A_{1}^{\prime}$ is g-bounded, for some $g \in \mathcal{K}^{\prime} \backslash\{0\}$;

hold. It follows that $r\left(A_{2}\right)<r\left(A_{1}\right)$ if, and only if, there exist $\gamma \in(0,1)$ and $N \in \mathbb{N}$ such that

$$
\left(A_{2}^{\prime}\right)^{n} f_{1} \leq\left(\gamma r_{1}\right)^{n} f_{1} \quad \forall n \in \mathbb{N}, n \geq N
$$


A drawback of Lemmas 3.1, 3.2 and 3.3 is the requirement that the spectral radius of $A_{2}$ or $A_{1}$ is an eigenvalue with positive eigenvector or eigenfunctional, respectively. Our next lemma relaxes that requirement for $u$-upper bounded operators. We recall that every bounded positive operator with respect to a solid cone is $u$-bounded from above for any interior point $u$ of the cone, because $\mathcal{X}=\mathcal{X}_{u}$ for such $u$. The proof is the same as one direction of Lemma 3.2, and so is omitted.

Lemma 3.4 Let $\mathcal{X}$ denote a real Banach space, with a reproducing and normal cone $\mathcal{K} \subset \mathcal{X}$ and positive linear operators $A_{1}, A_{2} \in \mathcal{B}(\mathcal{X})$ which satisfy $A_{2} \leq A_{1}$ and $A_{1} \neq A_{2}$. Assume that $A_{1}$ is $u$-bounded from above, for some $u \in \mathcal{K} \backslash\{0\}$. If there exist $\gamma \in(0,1)$ and $N \in \mathbb{N}$ such that

$$
A_{2}^{n} u \leq \gamma^{n} A_{1}^{n} u \quad \forall n \in \mathbb{N}, n \geq N,
$$

then $r\left(A_{2}\right)<r\left(A_{1}\right)$.

Finally, Lemma 3.4 may be formulated for the adjoint operator as well, mutatis mutandis, and so we do not give a formal statement.

\section{Strict monotonicity of spectral radii by spectral theory}

Here we derive sufficient conditions for (1.1) in terms of the operators $A_{1}, A_{2}$ and the cone $\mathcal{K} \subset \mathcal{X}$ which avoid checking estimates of the form (3.1), (3.2) or (3.3). To that end, we formulate the sequential assumptions:

(A.1) There exists $w_{1} \in \mathcal{K}, w_{1} \neq 0$, such that $A_{1} w_{1} \leq r\left(A_{1}\right) w_{1}$.

(A.2) There exists $f_{2} \in \mathcal{K}^{\prime}, f_{2} \neq 0$ such that $A_{2}^{\prime} f_{2} \geq r\left(A_{2}\right) f_{2}$.

(A.3) $f_{2}(y)>0$ where $y:=\left(A_{1}-A_{2}\right) w_{1} \in \mathcal{K}$.

Note that $y \in \mathcal{K}$ in (A.3) follows as $w_{1} \in \mathcal{K}$ and by our standing assumption that $A_{2} \leq A_{1}$.

Clearly, a necessary condition for (A.3) is that $y \neq 0$, that is, the operators $A_{1}, A_{2}$ and $w_{1}$ satisfy

$$
A_{2} w_{1} \neq r_{1} w_{1}
$$

The assumptions (A.1)-(A.3) are sufficient for strict monotonicity of the spectral radii, recorded in our main result.

Theorem 3.5 Let $\mathcal{X}$ denote a real Banach space, with a cone $\mathcal{K} \subset \mathcal{X}$ and positive linear operators $A_{1}, A_{2} \in \mathcal{B}(\mathcal{X})$ which satisfy $A_{2} \leq A_{1}$ and $A_{1} \neq A_{2}$. If (A.1)-(A.3) are satisfied, then $r\left(A_{2}\right)<r\left(A_{1}\right)$.

Proof Combining (A.1)-(A.3), we see that

$$
0<f_{2}(y)=f_{2}\left(\left(A_{1}-A_{2}\right) w_{1}\right) \leq f_{2}\left(\left(r_{1} I-A_{2}\right) w_{1}\right) \leq\left(r_{1}-r_{2}\right) f_{2}\left(w_{1}\right)
$$

from which we conclude that $r_{1}>r_{2}$, as required. 
We proceed to gather sufficient conditions for (A.1)-(A.3) and (3.5) to hold, formulated as the following three lemmas. We do not claim that the following lists are exhaustive. Obviously, (A.1) holds (with equality) if the spectral radius of $A_{1}$ is an eigenvalue of $A_{1}$, with associated positive eigenvector $w_{1} \in \mathcal{K}$. This is the approach we take. Although it is known that under mild assumptions the spectral radius belongs to the spectrum of a positive operator, it need not be an eigenvalue in general. Akin to (A.1), assumption (A.2) is satisfied with equality if $A_{2}$ admits a positive eigenfunctional corresponding to the spectral radius - a positive left eigenvector in the finite-dimensional case. We highlight that assumptions (A.1) and (A.2) are themselves not sufficient for (1.1), as the counter-example (1.3) demonstrates. Thus, the third assumption (A.3) is crucial and is a coupling condition between $A_{1}$ and $A_{2}$ where strict positivity plays a role.

Lemma 3.6 Imposing the notation of Theorem 3.5, if any one of the following:

(a) $\mathcal{X}=\mathbb{R}^{N}$, for some $N \in \mathbb{N}$;

(b) $\mathcal{K}$ is reproducing, $A_{1}^{k}$ is compact for some $k \in \mathbb{N}$, with $r\left(A_{1}\right)>0$;

(c) $\mathcal{K}$ is reproducing, normal and minhedral, $A_{1}$ is monotonically compact and $u$ bounded;

(d) $\mathcal{K}$ is reproducing and normal, $A_{1}$ is focussing, non-degenerate and $u$-bounded;

(e) $\mathcal{K}$ is reproducing and normal, $A_{1}$ is Riesz with respect to $\mathcal{C}(\mathcal{X})$, with $r\left(A_{1}\right)>0$;

are satisfied, then (A.1) holds.

We recall that a positive cone $\mathcal{K}$ is minhedral if every finite subset of $\mathcal{X}$ which is bounded with respect to the partial order induced by $\mathcal{K}$ has a supremum. The reader is referred as well to [12, Theorems 9.8,9.9] for the special cases that $A_{1}$ is an integral operator. Further, recall that an element $a$ of a Banach algebra $\mathcal{A}$ is called Riesz with respect to a closed ideal $\mathcal{I}$ if the spectrum of the element $a+\mathcal{I}$ in the quotient algebra $\mathcal{A} / \mathcal{I}$ is zero, see [20] or [2].

Proof of Lemma 3.6 (a) See [12, Theorem 9.1, p.87].

(b) See [12, Theorem 9.3, p.87].

(c) See [12, Theorem 9.7, p.92].

(d) See [12, Theorem 10.2, p. 105] and the second, unnamed, result in [12, Section 11.4 , p. 115].

(e) It follows from [3, Theorem 1.7.3] that $\mathcal{K}$ is reproducing and normal if, and only if, the cone of positive operators is normal in the Banach algebra $\mathcal{B}(\mathcal{X})$. This cone is a closed algebra cone, see [20], which is semi-simple. The set of compact operators $\mathcal{C}(\mathcal{X})$ is readily shown to be a closed, inessential ideal of $\mathcal{B}(\mathcal{X})$. Thus, the claim follows by [20, Theorem 3.7] which gives that there exists nonzero, positive $U \in \mathcal{B}(\mathcal{X})$ such that $A_{1} U=r\left(A_{1}\right) U$. Taking $v \in \mathcal{K}$ such that $w_{1}:=U v \neq 0$ yields (A.1).

The next lemma contains sufficient conditions for (A.2) and (A.3).

Lemma 3.7 Imposing the notation of Theorem 3.5, any one of the following:

(f) there exists $m \in \mathbb{N}$ such that $A_{2}^{m}$ is compact;

(g) $\mathcal{K}$ is normal and reproducing and $A_{2}$ is Riesz with respect to $\mathcal{C}(\mathcal{X})$, with $r\left(A_{2}\right)>$ 0 ; 
(h) $\mathcal{K}$ is normal and solid;

(i) $\mathcal{K}$ is reproducing and normal, and $A_{2}$ is u-bounded;

is sufficient for (A.2). Define $y:=\left(A_{1}-A_{2}\right) w_{1}$. If, in addition to one of $(f)-(i)$ above, (A.1), (3.5) and any one of the following:

(j) $y$ is a quasi-interior point of $\mathcal{K}$;

(k) $A_{2}$ is irreducible;

(1) $A_{2}$ is u-bounded;

hold, then (A.3) is satisfied.

Proof (f) The claim follows from [12, Theorem 9.2, p. 87] applied to $\mathcal{A}^{\prime} \in \mathcal{B}\left(\mathcal{X}^{\prime}\right)$, where we have used that $A^{\prime}$ is compact if $A$ is, Schauder's Theorem (see, for example, [14, Theorem 7, p.243]).

(g) If $A_{2}$ is Riesz with respect to the compact linear operators $\mathcal{C}(\mathcal{X})$, then $A_{2}^{\prime} \in$ $\mathcal{B}\left(\mathcal{X}^{\prime}\right)$ is Riesz with respect to the compact linear operators $\mathcal{C}\left(\mathcal{X}^{\prime}\right)$, and the claim follows from [20, Theorem 3.7] applied to $A_{2}^{\prime}$. Here we have used that $\mathcal{K}$ is a normal and reproducing cone implies that $\mathcal{K}^{\prime}$ is as well (see [12, Theorems 4.5, 4.6, p. 40]), and so from [3, Theorem 1.7.3] the cone of positive operators $\mathcal{X}^{\prime} \rightarrow \mathcal{X}^{\prime}$ is normal in the semi-simple Banach algebra $\mathcal{B}\left(\mathcal{X}^{\prime}\right)$.

(h) and (i) The claim follows from [12, Theorem 9.12, pp. 99-100].

That any of (j)-(1) and (A.1) and (3.5) are sufficient for (A.3) follows from [12, Theorem 16.3, p. 171], once we notice from (A.1) that

$$
A_{2} w_{1} \leq A_{1} w_{1} \leq r_{1} w_{1}
$$

We next provide sufficient conditions for the inequality (3.5) to hold.

Lemma 3.8 Imposing the notation of Theorem 3.5, let $u \in \mathcal{K} \backslash\{0\}$ and assume that (A.1) holds. The conditions:

(m) $\mathcal{X}=\overline{\mathcal{K}-\mathcal{K}}$ (for example, $\mathcal{K}$ is reproducing) and $w_{1}$ is a quasi-interior point of $\mathcal{K}$;

(n) $\mathcal{K}$ is reproducing, $A_{1}$ is $u$-bounded and $\left(A_{1}-A_{2}\right)^{2} \neq 0$;

(p) $\mathcal{K}$ is reproducing, $A_{1}$ is $u$-bounded and there exists $x \in \mathcal{X}$ such that $-n w_{1} \leq$ $x \leq n w_{1}$ for some $n \in \mathbb{N}$ with the property that $\left(A_{1}-A_{2}\right) x \neq 0$;

are each sufficient for (3.5).

We note that irreducibility of $A_{2}$ is sufficient for irreducibility of $A_{1}$ which in turn is sufficient for irreducibility of $A_{1}+A_{2}$. If this latter condition holds, then, in light of (A.1) and the estimates

$$
\left(A_{1}+A_{2}\right) w_{1} \leq 2 A_{1} w_{1} \leq 2 r_{1} w_{1},
$$

it follows that $w_{1}$ is a quasi-interior point of $\mathcal{K}$. 
Proof of Lemma $3.8(\mathrm{~m})$ Choose $x^{*} \in \mathcal{K}$ such that $w^{*}:=\left(A_{1}-A_{2}\right) x^{*} \in \mathcal{K} \backslash\{0\}$. Thus, by [12, Theorem 2.2, pp. 20-21], there exists a positive functional $g \in \mathcal{K}^{\prime}$ such that $g\left(w^{*}\right)>0$. Therefore, $h \in \mathcal{K}^{\prime}$ defined by

$$
h:=g \circ\left(A_{1}-A_{2}\right): \mathcal{K} \rightarrow \mathcal{K} \rightarrow \mathbb{R}_{+},
$$

satisfies

$$
h\left(x^{*}\right)=g\left(\left(A_{1}-A_{2}\right) x^{*}\right)=g\left(w^{*}\right)>0,
$$

and so $h$ is non-zero. As $w_{1}$ is a quasi-interior point, and by (A.1),

$$
0<h\left(w_{1}\right)=g\left(\left(A_{1}-A_{2}\right) w_{1}\right)=g(y),
$$

whence $y \neq 0$.

(n) In Lemma 3.2 we proved the inequality (3.4), that $u$-boundedness of $A_{1}$ implies that $A_{1}$ is $w_{1}$-bounded from above. Clearly, $A_{1}-A_{2}$ is $w_{1}$-bounded from above as well.

Next, the reproducing property of $\mathcal{K}$ implies that there exists $x^{*} \in \mathcal{K}$ such that

$$
y^{*}=\left(A_{1}-A_{2}\right)^{2} x^{*} \in \mathcal{K} \backslash\{0\},
$$

so that clearly both $x^{*} \neq 0$ and $\gamma^{*}:=\gamma\left(\left(A_{1}-A_{2}\right) x^{*}\right)>0$. Thus, invoking the $w_{1}$-upper boundedness of $A_{1}-A_{2}$ with $x=x^{*}$,

$$
0 \leq\left(A_{1}-A_{2}\right) x^{*} \leq \gamma\left(\left(A_{1}-A_{2}\right) x^{*}\right) w_{1}=\gamma^{*} w_{1},
$$

and applying $A_{1}-A_{2}$ to both sides yields that

$$
y^{*}=\left(A_{1}-A_{2}\right)^{2} x^{*} \leq \gamma^{*}\left(A_{1}-A_{2}\right) w_{1} \leq \gamma^{*} y .
$$

Since $y^{*} / \gamma^{*} \neq 0$, we conclude that $y \neq 0$.

(p) Similarly to (n), $A_{1}-A_{2}$ is $w_{1}$-bounded from above. Thus, $\left.\left(A_{1}-A_{2}\right)\right|_{\mathcal{X}_{w_{1}}} \in$ $\mathcal{B}\left(\mathcal{X}_{w_{1}}\right)$ and, by assumption, is not equal to the zero operator. Therefore, invoking (2.2) and (ii), we see that

$$
0<\left\|\left(A_{1}-A_{2}\right) \mid \mathcal{X}_{w_{1}}\right\|_{w_{1}}=\left\|\left(A_{1}-A_{2}\right) w_{1}\right\|_{w_{1}}=\|y\|_{w_{1}},
$$

demonstrating that $y \neq 0$.

To summarise briefly, Lemmas 3.7 and 3.8 place assumptions on $A_{2}$ which, via Theorem 3.5, ensure that (1.1) holds. Our final result is inspired by [19, Theorem 4.3], and instead places more assumptions on $A_{1}$.

Proposition 3.9 Let $\mathcal{X}$ denote a real Banach space, with reproducing and normal cone $\mathcal{K} \subset \mathcal{X}$ which induces a Riesz space, and positive linear operators $A_{1}, A_{2} \in \mathcal{B}(\mathcal{X})$ which satisfy $A_{2} \leq A_{1}$ and $A_{1} \neq A_{2}$. If there exist 
(I) $w_{1} \in \mathcal{K} \backslash\{0\}$ such that $A_{1} w_{1}=r_{1} w_{1}$;

(II) $f_{1}, f_{2} \in \mathcal{K}^{\prime} \backslash\{0\}$ such that $A_{i}^{\prime} f_{i}=r_{i} f_{i}$;

and one of:

(III) (1) $A_{1}$ is $u$-bounded, for some $u \in \mathcal{K} \backslash\{0\}$, a quasi-interior point of $\mathcal{K}$;

(2) $A_{1}$ is irreducible;

hold, then $r\left(A_{2}\right)<r\left(A_{1}\right)$.

Recall that an ordered Banach space $\mathcal{X}$ is a Riesz space if for each $u, v \in \mathcal{X}$, the supremum and infimum of $u$ and $v$ (with respect to the ordering induced by the cone $\mathcal{K}$ in this instance) also are elements of $\mathcal{X}$. Riesz spaces are well-studied objects; see, for example [1, p. 2] or [18, p. 48].

With reference to assumption (III) (1), in light of the inequalities

$$
\alpha\left(w_{1}\right) u \leq A_{1} w_{1}=r_{1} w_{1} \leq \beta\left(w_{1}\right) u,
$$

it follows that $u$ is a quasi-interior point of $\mathcal{K}$ if, and only if, $w_{1}$ is.

Proof of Proposition 3.9 The assumption that $\mathcal{K}$ is normal and reproducing implies that $r_{2} \leq r_{1}$ by, for example, [5, Theorem 1.1]. Seeking a contradiction, assume that $r_{2}=r_{1}=: r$. If $A_{1}$ is irreducible, then $w_{1}$ is quasi-interior point, and as for all $x \in \mathcal{K}$, $x \neq 0$

$$
0<f_{1}\left(A_{1}^{n} x\right)=r^{n} f_{1}(x)
$$

for some $n \in \mathbb{N}$ by [12, Theorem 11.2, p.113], we conclude that $r>0$ and $f_{1}$ is strictly positive. Alternatively, if $A_{1}$ is $u$-bounded by a quasi-interior point, then $f_{1}(u)>0$. It now follows from (2.1) that

$$
0<\alpha(x) f_{1}(u) \leq f_{1}\left(A_{1} x\right)=r f_{1}(x) \quad \forall x \in \mathcal{K} \backslash\{0\}
$$

meaning $r>0$ and $f_{1}$ is strictly positive. Using (II), we now estimate that

$$
r f_{2}=A_{2}^{\prime} f_{2} \leq A_{1}^{\prime} f_{2} \text {. }
$$

Since $\phi:=A_{1}^{\prime} f_{2}-r f_{2} \in \mathcal{K}^{\prime}$, and $w_{1}$ is a quasi-interior point, the equality

$$
\phi\left(w_{1}\right)=f_{2}\left(A_{1} w_{1}\right)-r f_{2}\left(w_{1}\right)=(r-r) f_{2}\left(w_{1}\right)=0,
$$

implies that $\phi=0$, that is, $A_{1}^{\prime} f_{2}=r f_{2}$.

We claim that

$$
f_{2}=c f_{1},
$$

for some $c>0$. The arguments which follow are based on those of [12, pp.112-113]. To that end, consider $g:=t f_{1}-f_{2} \in \mathcal{X}^{\prime}$, for $t>0$. If $g=0$ for some $t>0$, then there is nothing to prove. We consider two exhaustive possibilities. Either we may choose $t>0$ sufficiently large such that $g \in \mathcal{K}^{\prime} \backslash\{0\}$ and $g(x)=0$ for some $x \in \mathcal{K} \backslash\{0\}$ 
or, for these $t>0, g \notin \mathcal{K}^{\prime} \cup\left(-\mathcal{K}^{\prime}\right)$. In the first case we reach the contradiction that the obvious equality $A_{1}^{\prime} g=r g$ implies that $g$ must be strictly positive. In the second, the element $g_{+}:=\sup \{0, g\} \in \mathcal{K}^{\prime}$ is well-defined by the minhedrality of $\mathcal{K}^{\prime}$, see [12, Theorem 6.4, p. 61] and is not strictly positive. Moreover, as $A_{1}^{\prime} g_{+} \geq 0$ and $A_{1}^{\prime} g_{+} \geq r g$, it follows that $A_{1}^{\prime} g_{+} \geq r g_{+}$, yet $A_{1}^{\prime} g_{+} \neq r g_{+}$(else $g_{+}$would be strictly positive). Therefore, on the one hand, the functional

$$
h:=\sum_{k=0}^{\infty}\left(2\left\|A^{\prime}\right\|\right)^{-k}\left(A_{1}^{\prime}\right)^{k}\left(A_{1}^{\prime} g_{+}-r g_{+}\right) \in \mathcal{K}^{\prime} \backslash\{0\},
$$

satisfies

$$
h\left(w_{1}\right)=\sum_{k=0}^{\infty}\left(2\left\|A^{\prime}\right\|\right)^{-k}\left(A_{1}^{\prime}\right)^{k} \underbrace{\left(g_{+}\left(A_{1} w_{1}\right)-r g_{+}\left(w_{1}\right)\right)}_{=0}=0 .
$$

However, on the other hand, the easily established estimate

$$
A_{1}^{\prime} h \leq 2\left\|A_{1}^{\prime}\right\| h
$$

implies that $h$ is strictly positive, and so $h\left(w_{1}\right)>0$, a contradiction. We have established (3.7), and so $f_{2}$ is also strictly positive, as $f_{1}$ is.

Let $v \in \mathcal{K} \backslash\{0\}$ be such that $\left(A_{1}-A_{2}\right) v \neq 0$, so that $f_{i}(v)>0$, as $v \neq 0$. Thus, we arrive at the contradiction

$$
\begin{aligned}
r & =r_{1}=\frac{f_{1}\left(A_{1} v\right)}{f_{1}(v)}=\frac{f_{2}\left(A_{1} v\right)}{f_{2}(v)}=\frac{f_{2}\left(A_{2} v\right)}{f_{2}(v)}+\frac{f_{2}\left(\left(A_{1}-A_{2}\right) v\right)}{f_{2}(v)} \\
& =r_{2}+\frac{f_{2}\left(\left(A_{1}-A_{2}\right) v\right)}{f_{2}(v)}>r .
\end{aligned}
$$

We conclude this section with some commentary, first on the assumptions of Proposition 3.9, and then make some comparisons with [19].

Remark 3.10 Inspection of the proof of Proposition 3.9 shows where the assumptions made are applied, and how these may be substituted. The assumption that $\mathcal{K}$ is normal and reproducing is used to ensure that $r_{2} \leq r_{1}$, from which a contradiction argument is used. If $\mathcal{X}=\overline{\mathcal{K}-\mathcal{K}}$ and either $A_{2}$ is compact or $r\left(A_{2}\right)$ is a pole of the resolvent of (the complexification of) $A_{2}$, then $r\left(A_{2}\right) \leq r\left(A_{1}\right)$, see [5, Theorem 1.2, Corollary 1.3]. The Riesz property and normality are together used in the proof of (3.7) to ensure that $\mathcal{K}^{\prime}$ is minhedral, and so the element $g_{+}=\sup \{g, 0\}$ is well-defined. If the cone is $\mathcal{K}^{\prime}$ is solid, then a different argument may be used to establish (3.7), see [12, Theorem 11.1]. By [12, Theorems 5.6 and 5.10], solidity of $\mathcal{K}^{\prime}$ is equivalent to the existence of a uniformly positive functional $f \in \mathcal{K}^{\prime}$, that is, there exists $\theta>0$ such that $f(x) \geq \theta\|x\|$ for all $x \in \mathcal{K}$. Irreducibility or $u$-boundedness is used to establish 
that $f_{1}$ is strictly positive and that (3.7) holds, so that $f_{2}$ is strictly positive as well. Strict positivity is required so that the arguments in (3.8) make sense.

Remark 3.11 There is overlap between our results and those of [19], namely [19, Theorems 4.3, 4.4]. In both of these results the cone $\mathcal{K}$ is assumed closed, and so the cones in [19, Theorems 4.3, 4.4] are assumed reproducing (although that assumption is not made throughout [19]). Our Proposition 3.9, including its proof, is based on [19, Theorem 4.3], and strengthens it slightly by permitting that $A_{1}$ is $u$-bounded. Irreducibility as used here goes by the term semi-nonsupporting operator in [19], and is assumed of $A_{1}$ in [19, Theorem 4.3], but the concepts are equivalent via [12, Theorem 11.2, p. 113].

Theorem 3.5 is comparable with, but generalises, [19, Theorem 4.4], where it is assumed that $A_{1}$ is strongly positive (non-zero positive elements are mapped to quasipositive ones), which ensures that (1) holds. Finally, the assumption that $\left(A_{1}-A_{2}\right) x$ is a quasi-interior point whenever $x \in \mathcal{K} \backslash\{0\}$ is stronger than our assumption (i). Finally, we do note that [19, Theorem 4.4] proves other assertions than solely (1.1).

\section{Examples}

Example 4.1 Let $\mathcal{X}=C([0,1])$ denote the Banach space of continuous real-valued functions $[0,1] \rightarrow \mathbb{R}$ equipped with the supremum norm, and let $\mathcal{K}$ denote the cone of nonnegative-valued functions. This cone is solid (and so reproducing) and normal. The operator

$$
A_{2}: \mathcal{X} \rightarrow \mathcal{X}, \quad\left(A_{2} x\right)(t)=t x(t) \quad \forall x \in \mathcal{X}, \forall t \in[0,1]
$$

is linear, continuous and positive. Evidently, for $x \in \mathcal{X}$ with $\|x\|_{\infty}=1$

$$
\left\|A_{2} x\right\|_{\infty}=\sup _{t \in[0,1]}|t x(t)| \leq \sup _{t \in[0,1]}|x(t)|=\|x\|_{\infty}=1
$$

and the bound is achieved when $x \equiv 1$, so $\left\|A_{2}\right\|_{\infty}=1$. Moreover, $\left\|A_{2}^{n}\right\|=1$ for all $n \in \mathbb{N}$, and so $r\left(A_{2}\right)=1$. However, it is clear that $A_{2} x=\lambda x$ has no non-zero solutions, and so $A_{2}$ has no eigenvalues and eigenvectors. For $\rho>1$ and $\omega>0$ define

$$
A_{1}: \mathcal{X} \rightarrow \mathcal{X}, \quad\left(A_{1} x\right)(t)=t(\rho+(\rho-1) \sin (\omega t)) x(t) \quad \forall x \in \mathcal{X}, \forall t \in[0,1],
$$

which is also linear, bounded and positive. The coefficients of $x$ in $A_{1}$ and $A_{2}$ are plotted in Fig. 1, which visualises the readily established properties that $A_{2} \leq A_{1}$ and $A_{2} \neq A_{1}$. As with $A_{2}, A_{1}$ does not have any eigenvalues, but noting that for all $s \in[0,1], g_{s} \in \mathcal{K}^{\prime}$ defined by $g_{s}(x)=x(s)$ clearly satisfies

$$
\begin{aligned}
& \qquad \begin{array}{l}
\left(A_{2}^{\prime} g_{s}\right)(x)=s x(s)=s g_{s}(x) \\
\text { and } \quad\left(A_{1}^{\prime} g_{s}\right)(x)=s \theta(s) x(s)=s \theta(s) g_{s}(x),
\end{array}
\end{aligned}
$$


Fig. 1 Coefficient of $A_{2}$ dashed line and coefficient of $A_{1}$, solid line. Here $\rho=1.1$ and $\omega=40 \pi$

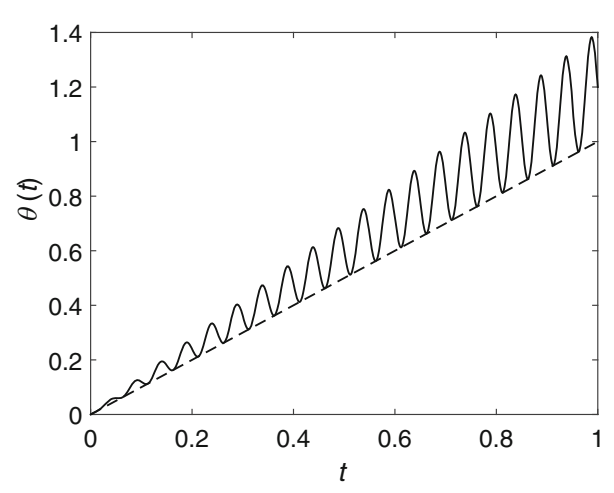

where $\theta:[0,1] \rightarrow \mathbb{R}_{+}$is given by

$$
\theta(t):=\rho+(\rho-1) \sin (\omega t) \quad \forall t \in[0,1],
$$

we see that both $A_{1}^{\prime}$ and $A_{2}^{\prime}$ have positive eigenfunctionals.

For ease of exposition, assume that $\theta(1)>1$ and choose $\rho \in(1 / \theta(1), 1)$. In this case, we have that

$$
A_{2}^{\prime} g_{1}=g_{1} \leq \rho \theta(1) g_{1}=\rho A_{1}^{\prime} g_{1}
$$

An application of Lemma 3.1 yields that $r\left(A_{2}\right)=1<r\left(A_{1}\right)$.

Example 4.2 Let $\mathcal{X}=\mathbb{R}^{N}$ for some $N \in \mathbb{N}, \mathcal{K}=\mathbb{R}_{+}^{N}$ which induces the partial order of componentwise inequality. Let $A_{1}, A_{2} \in \mathbb{R}_{+}^{N \times N}$ denote nonnegative matrices. If $A_{2} \leq A_{1}, A_{2} \neq A_{1}$ and $A_{2}$ is irreducible, then $r\left(A_{2}\right)<r\left(A_{1}\right)$. The claim is known from [22, Theorem 9], but in the present context follows from Theorem 3.5, after noting that assumptions (a), (h), (j) and (m) are satisfied. If $A_{1}$ is irreducible, then $r\left(A_{2}\right)<r\left(A_{1}\right)$ is known from [4, Corollary 1.5, p.27], but in the present setting follows from Proposition 3.9.

In [4, Corollary 1.5, p.27], it is proven that irreducibility of $A_{1}+A_{2}$ is sufficient for $r\left(A_{2}\right)<r\left(A_{1}\right)$, which is seemingly weaker than requiring $A_{1}$ is irreducible. We comment that there is no generality gained by assuming that $\alpha A_{1}+(1-\alpha) A_{2}$ is irreducible or $u$-bounded, for some $\alpha \in(0,1]$, at least under our standing assumption that $A_{2} \leq A_{1}$, even in more general operator settings. Indeed, the trivially established inequalities

$$
\alpha A_{1} \leq \alpha A_{1}+(1-\alpha) A_{2} \leq A_{1},
$$

shows that $\alpha A_{1}+(1-\alpha) A_{2}$ is irreducible or $u$-bounded if, and only if, $A_{1}$ is-where Proposition 3.9 applies.

Example 4.3 Let $\mathcal{X}=L^{p}(\Omega ; \mathbb{R})$ for $1 \leq p<\infty$ and where $\Omega$ is a compact metric space. Let $\mathcal{K}_{+}$denote the cone of functions $\Omega \rightarrow \mathbb{R}$ which are nonnegative almost 
everywhere on $\Omega$, which is reproducing and normal, but not solid. Consider $A_{1}, A_{2} \in$ $\mathcal{B}(\mathcal{X})$ defined by

$$
\left(A_{i} x\right)(t)=\int_{\Omega} k_{i}(t, s) x(s) d s, \quad i \in\{1,2\},
$$

for some kernels $k_{i}: \Omega \times \Omega \rightarrow \mathbb{R}$. The operators $A_{i}$ are positive if

$$
k_{i}(t, s) \geq 0 \text { for almost all }(t, s) \in \Omega \times \Omega \text {, }
$$

and moreover, by [12, Theorem 2.1, p.19], nonnegativity of the kernel (4.1) is sufficient for $A_{i}$ to be bounded. By linearity, the inequality $A_{2} \leq A_{1}$ is equivalent to

$$
k_{2}(t, s) \leq k_{1}(t, s) \text { for almost all }(t, s) \in \Omega \times \Omega,
$$

and $A_{2} \neq A_{1}$ means that there exist sets of positive measure $\Omega_{i}^{*} \subset \Omega$ such that

$$
k_{2}(t, s)<k_{1}(t, s) \text { for almost all }(t, s) \in \Omega_{1}^{*} \times \Omega_{2}^{*} \text {. }
$$

It is well-known that integral operators are compact under rather general assumptions on the kernel. If $k_{1} \in L^{1}(\Omega \times \Omega)$, then $A_{1}$ is compact and so is $A_{2}$ by (4.2). It follows from (b) and (e) that (A.1) and (A.2) hold. The operators $A_{i}$ are $u_{i}$-bounded if there exists $u_{i} \in \mathcal{K}, u_{i} \neq 0$ and nonnegative functions $\alpha_{i}, \beta_{i} \in L^{q}(\Omega)$, where $q \in(1, \infty)$ is complimentary to $p$, such that

$$
\alpha_{i}(s) u_{i}(t) \leq k_{i}(t, s) \leq \beta_{i}(s) u_{i}(t) \text { for almost all }(t, s) \in \Omega \times \Omega .
$$

The operators $A_{i}$ are irreducible if, for any measurable proper subset $\Omega_{1} \subset \Omega$ with positive measure, there exist closed sets $\Gamma_{1} \subset \Omega$ and $\Gamma_{2} \subset \Omega \backslash \Omega_{1}$ such that

$$
k_{i}(t, s)>0 \text { for almost all }(t, s) \in \Gamma_{1} \times \Gamma_{2} .
$$

Irreducibility of $A_{2}(\mathrm{k})$ is sufficient for (A.3), as is $u$-boundedness of $A_{2}$ (1) and $w_{1}$ a quasi-interior point (m). Alternatively, if one of (j)-(l) hold and $\Omega^{*}:=\Omega_{1}^{*} \cap \Omega_{2}^{*}$ has positive measure, then there exists $x \in \mathcal{K}$ such that $\left(A_{1}-A_{2}\right)^{2} x \neq 0$, and so statement $(\mathrm{m})$ holds, which together are sufficient for (A.3). To see that (m) holds, we compute that for $x \in \mathcal{K}$ and almost all $t \in \Omega$

$$
\begin{aligned}
\left(A_{1}-A_{2}\right)^{2} x(t) & =\int_{s \in \Omega}\left(k_{1}-k_{2}\right)(t, s)\left(A_{1}-A_{2}\right) x(s) d s \\
& =\int_{s \in \Omega}\left(k_{1}-k_{2}\right)(t, s) \int_{\tau \in \Omega}\left(k_{1}-k_{2}\right)(s, \tau) x(\tau) d \tau d s \\
& =\iint_{s, \tau \in \Omega} \underbrace{\left(k_{1}-k_{2}\right)(t, s)\left(k_{1}-k_{2}\right)(s, \tau)}_{>0 \text { on } \Omega^{*} \times \Omega^{*}} x(\tau) d \tau d s .
\end{aligned}
$$


Example 4.4 Consider the discrete-time switched dynamical system

$$
x(t+1)=\mathcal{B}(t) x(t) \quad x(0)=x^{0} \quad t \in\{0\} \cup \mathbb{N},
$$

where $\mathcal{B}(t) \in\left\{B_{1}, \ldots, B_{N}\right\}$ for each $t \in\{0\} \cup \mathbb{N}$ and given positive $B_{k} \in \mathcal{B}(\mathcal{X})$. Switched systems are a popular and important class of control systems which, for example, arise in dynamical systems with several distinct modes of operation. Their interest is in part motivated by the property that even if $r\left(B_{k}\right)<1$ for every $k$, the zero equilibrium of (4.3) may be unstable, depending on the switching between the $B_{k}$, captured via $\mathcal{B}$. We refer the reader to the survey articles [15] or [16] and the references therein, for further background on switched systems. Much attention has been devoted in the systems and control literature to the stability of switched positive dynamical systems, particularly by Valcher, Shorten and their collaborators; see, for instance $[9,11]$.

Here we simply record an elementary application of our results. Namely, if $B_{1}, \ldots, B_{N} \leq A_{2} \leq A_{1}$, with $A_{1} \neq A_{2}, r\left(A_{1}\right)=1$ and (1.1) holds, then $r\left(A_{2}\right)<1$ and the zero equilibrium is globally exponentially stable if the cone is normal. Indeed, the solution $x$ of (4.3) satisfies

$x(t) \leq A_{2}^{t} x(0) \Rightarrow 0 \leq\|x(t)\| \leq\left\|A_{2}^{t}\right\| \cdot\|x(0)\| \leq M r_{2}^{t}\|x(0)\| \rightarrow 0$ as $t \rightarrow \infty$,

for some $M>0$ by, for example, [17, Lemma 1].

Open Access This article is distributed under the terms of the Creative Commons Attribution 4.0 International License (http://creativecommons.org/licenses/by/4.0/), which permits unrestricted use, distribution, and reproduction in any medium, provided you give appropriate credit to the original author(s) and the source, provide a link to the Creative Commons license, and indicate if changes were made.

\section{References}

1. Aliprantis, D.C., Burkinshaw, O.: Positive Operators. Springer, New York (2006)

2. Barnes, B.A., Murphy, G.J., Smyth, M.R.F., West, T.T.: Research Notes in Mathematics 67. Riesz and Fredholm Theory in Banach Algebras. Pitman, Bath (1982)

3. Batty, C.J., Robinson, D.W.: Positive one-parameter semigroups on ordered Banach spaces. In: Bratteli, O., Jørgensen, P.E.T. (eds.) Positive Semigroups of Operators, and Applications, pp. 221-296. Springer, Dordrecht (1984)

4. Berman, A., Plemmons, R.J.: Nonnegative Matrices in the Mathematical Sciences. SIAM, Philadelphia (1994)

5. Burlando, L.: Monotonicity of spectral radius for positive operators on ordered Banach spaces. Arch. der Math. 56, 49-57 (1991)

6. Deimling, K.: Nonlinear Functional Analysis. Springer, Berlin (1985)

7. Easterling, M., Ellner, S., Dixon, P.: Size-specific sensitivity: applying a new structured population model. Ecology 81, 694-708 (2000)

8. Ellner, S., Rees, M.: Integral projection models for species with complex demography. Am. Nat. 167, 410-428 (2006)

9. Fornasini, E., Valcher, M.E.: Stability and stabilizability criteria for discrete-time positive switched systems. IEEE Trans. Autom. Control 57, 1208-1221 (2012) 
10. Guiver, C., Mueller, M., Edholm, C., Rebarber, R., Jin, Y., Tenhumberg, B., Powell, J., Townley, S.: Simple adaptive control for positive linear systems with applications to pest management. SIAM J. Appl. Math. 76, 238-275 (2016)

11. Knorn, F., Mason, O., Shorten, R.: On linear co-positive Lyapunov functions for sets of linear positive systems. Automatica 45, 1943-1947 (2009)

12. Krasnosel'skij, M.A., Lifshits, J.A., Sobolev, A.V.: Positive Linear Systems. Heldermann, Berlin (1989)

13. Kreyszig, E.: Introductory Functional Analysis with Applications. Wiley, New York (1978)

14. Lax, P.D.: Functional Analysis. Wiley, New York (2002)

15. Liberzon, D., Morse, A.S.: Basic problems in stability and design of switched systems. IEEE Control Syst. Mag. 19, 59-70 (1999)

16. Lin, H., Antsaklis, P.J.: Stability and stabilizability of switched linear systems: a survey of recent results. IEEE Trans. Autom. Control 54, 308-322 (2009)

17. Logemann, H.: Stability and stabilizability of linear infinite-dimensional discrete-time systems. IMA J. Math. Control Inform. 9, 255-263 (1992)

18. Luxemburg, W.A.J., Zaanen, A.C.: Riesz Spaces, vol. I. North Holland Publishing Company, Amsterdam (1971)

19. Marek, I.: Frobenius theory of positive operators: comparison theorems and applications. SIAM J. Appl. Math. 19, 607-628 (1970)

20. Mouton, S., Raubenheimer, H.: More spectral theory in ordered banach algebras. Positivity 1, 305-317 (1997)

21. Muzundu, K.: Spectral theory in commutatively ordered Banach algebras. Ph.D. Thesis, Stellenbosch University, South Africa (2012)

22. Rheinboldt, W.C., Vandergraft, J.S.: A simple approach to the Perron-Frobenius theory for positive operators on general partially-ordered finite-dimensional linear spaces. Math. Comput. 27, 139-145 (1973)

23. Schaefer, H.: Some spectral properties of positive linear operators. Pac. J. Math. 10, 1009-1019 (1960) 